\title{
Quality Measurement for Mobile M-ERP Applications
}

\author{
Haeng-Kon Kim \\ School of Information Technology, Catholic University of Daegu, Korea \\ hangkon@cu.ac.kr
}

\begin{abstract}
Mobile business boosting the popularization of the M-ERP(Mobile ERP) in the enterprise: The high penetration of mobile phones enables each employee to become an effective information carrier of the enterprise. The information application of the enterprise becomes more clear-cut. The mobile M-ERP pivots on the frequently changing key indexes of the enterprise operation, and takes the personalized and format-based data collection, analysis and processing platform as tool. It consolidates and unifies quickly diverse people, internal data, and external applications of the enterprise into an integrated whole, and provides real-time, key, and overall data reports to the management personnel. The recent wave of enterprise resource planning (M-ERP) systems adoption is a significant commitment of resource and may affect almost all business processes. M-ERP systems are integrated systems in that they promote cooperation among groups, teamwork, and process expertise and business knowledge. Firm that successfully implements an M-ERP system should raise revenues or decrease costs. The main purpose of this paper is to investigate the relationship among M-ERP system internal control, quality and performance in Korean firms. To investigate the relationship, the questionnaires were collected from 131 M-ERP - adopting Korean manufacturing companies. The following results were determined by verifying six hypotheses using LISREL. Internal control support perceived quality and quality support perceived performance. We expect that the results of the research can be used as the guidance of the implementation strategy of M-ERP systems. And these results provide important insights that complement extant research findings and also raise future research issues.
\end{abstract}

Keyword: Mobile Enterprise Resource planning, Supply Chain Management, ERP Quality measurement, Mobile ERP Architecture

\section{Introduction}

Today, most business applications (e and m-business solutions) run either on a client server system, a web based system and mobile based systems have three main components: Enterprise Resource Planning (M-ERP), Supply Chain Management (SCM) and Customer Relationship Management (CRM). However, the main disadvantage of today's systems is that the workers, suppliers and customers have to be bound to their desktops in order to take advantage of these systems. There is a need to break this tethered, desktop model by extending the e-business solutions to the mobile workers. Mobile ebusiness solutions are the need of the hour, which are made possible today by advances in technology.

The recent wave of enterprise resource planning (M-ERP) systems adoption is a significant commitment of resource and may affect almost all business processes. Over the past decade, organizations have made significant investment in enterprise resource planning (M-ERP) systems. M-ERP referred to as enterprise -wide systems or enterprise systems, offer the seamless integration of all the information flowing through companyfinancial and accounting information. And M-ERP was one of the largest information 
technology (IT) environments in the past decades. But system integration has control weakness: data entry error is critical; decreasing data visibility; more complicated segregation duties etc. M-ERP is not a mere change in information system environment but an overall change in organizational structures and business processes. Therefore many firms experience a temporary decline in performance after M-ERP implementation [1, 2].

The most research on M-ERP focused on successful launching of M-ERP systems, such as critical success factors for M-ERP implementations. However, the study on the interrelation between the M-ERP system internal controls and organizational performance is extremely rare. Furthermore, it is not known if the study is actually performed, which examines the differential impacts of internal controls on the organizational performance between sound and weak internal control firms when M-ERP system is established. Research purpose of this study is to investigate the relationship among MERP system internal control, quality and performance [3].

In this paper, we study on the critical role of M-ERP systems' internal control, information quality and performance in the factory industry. This sets out the research model of influencing factors and the performance of the M-ERP system through theoretical studies based on Management Information Systems, and the empirically tests hypotheses related to the model.

\section{Related Work}

\subsection{Functions of Mobile M-ERP}

M-ERP system is comprehensive software packages that seek to integrate the complete range of business process and functions in order to present a holistic view of the business from a single information and information technology architecture [4, 5]. M-ERP systems can provide a wide array of benefits that are both tangible(e.g., reduced personnel, inventory, IT and procurement, transportation, and logistic costs; improved cash flow management, revenue, and profits) and intangible (e.g., increased visibility of corporate data, speed of decision making, and control over global business operations; improved customer responsiveness and business processes). There are several Mobile ERP functionalities to be equipped with the solutions as followings:

\section{- Early update of alarm information}

The alarm of the max. and min. inventory, the alarm of the delayed MOs and any other alarm messages will be sent to your mobile phone at the first moment possible. You do not need to worry about the loss caused due to an overlooked key event.

\section{-Subscribing enterprise management information - just like subscribing a paper}

This function can send you the expected business and report information at the specified time. You can be kept posted with the latest information of various aspects of the enterprise - just like skimming through a newspaper.

\section{- Reviewing and approving work flow at anytime and anywhere}

Employee vacation review and approving, PO review and approving and any other review and approving events, small and big alike, can be handled at anytime and anywhere. It goes free of the restriction of time and space. The fast response capability of the enterprise is improved.

\section{- Real-time query of information regardless of the location}

When you want to know about some data, you can do it with so much as an SMS, no matter where you are.

\section{-Implementing personalized information customization}

Self-defined personalized report, self-defined personalized bill review Traditional enterprise applications have been built around the wired networks and hence do not 
adequately address the need of extending the enterprise systems to the mobile employees and customers. However, now it is technologically possible to build mobile applications providing “anywhere, anytime” access to an organisation's employees and customers. The information required to do any job can be made available wherever it is required.

\subsection{Internal Control on M ERP}

Internal control on M ERP is a process -affected by an entity's board of directors, management, and other personnel- designed to provide reasonable assurance regarding the achievement of objectives in the following categories:

(1) reliability of financial reporting

(2) effectiveness and efficiency of operations

(3) compliance with applicable laws and regulations

Internal control research has addressed how auditors document or model internal controls separately from how they evaluate those controls [6]. Gadh et al., [7] presents a prototype model that can evaluate internal control systems based on its own evaluation rules and internal representation of those systems.

As Vasarhelyi [8] notes, information must be correct, in real-time, utilizing systems that are reliable by design and not by correcting the data after the fact. This paradigm shift emphasizes the internal control systems that govern the design, development, implementation, and monitoring of tourism information systems.

M-ERP helps companies control their purchasing, inventory, manufacturing, finance, and human resource activities by centralizing information collected from dispersed geographical sites [9]. [10] Examines whether organizing information about internal controls around business processes instead of control objectives produces stronger category knowledge during training and improves decision performance during internal control evaluation. They find that novices who were trained to evaluate internal controls using business-process-focused materials developed stronger category knowledge and identified more internal control issues during using a internal control evaluation task. Their findings suggest that the process focus may be a more effective framework for organizing internal control evaluation tasks as in Figure 1.

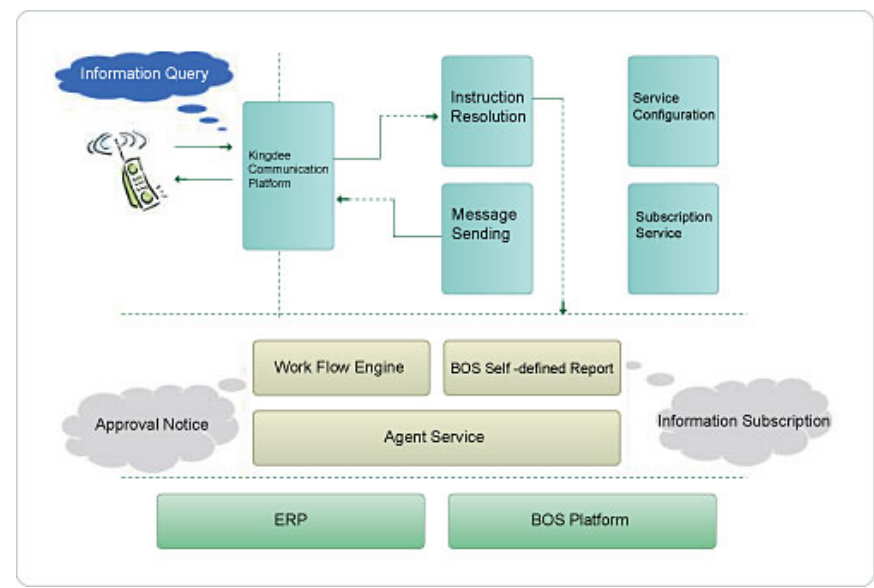

Figure 1. Overview of Architecture for Internal Control on M ERP

\subsection{ERP Quality}

To manage effectively, one must measure and analyze. To measure of M-ERP applications, however, one must define what to measure. Defining what Information Quality means, therefore, is crucial in managing information as a product. In the process of defining what information quality means, the participants will engage in problem 
identification and problem solving in the context of their own organizational setting. They must identify the necessary organizational processes and technical solutions for managing the information product.

The concept of information quality is more important than ever to business. Just as quality in goods/services has the capacity to satisfy people's needs. Information quality in decision-making has the capacity to satisfy internal and external customers. Hence, information quality has different implications under different decision dimensions. When M ERP applications quality measures activity becomes an essential organizational trend, it can provide internal and external customer service--where the service form is presentation of information and the content is the quality concept. The meaning of information quality, speaking on the basis of information systems concepts and purpose (data-information-decision-action), is that which achieves effective outcomes in making the right decisions. Decisions must be based on good, high-quality information, so information quality improvement is the critical element for M ERP applications quality measures. But it is difficult to evaluate the value of information quality; moreover, the evaluation must be based on the actions resulting from all kinds of decisions made by the information user. To manage effectively, one must measure and analyze. We have studies research opportunities in internal control quality and quality assurance. He discussed the importance of pursuing broad internal control quality and quality assurance research topics of wide practical and scholarly interest.

\subsection{CBD (Component-Based Development)}

Software components are binary units of independent production, acquisition, and deployment that interact to form a functioning system. Composite systems composed of software components are called component software [2, 3]. Abstractions, such as procedures, classes, modules, or even entire applications could form components, as long as they are in a 'binary' form that remains composable. The benefit of component software is as follows: components are the way to go because all other engineering disciplines introduced components as they became mature [6].

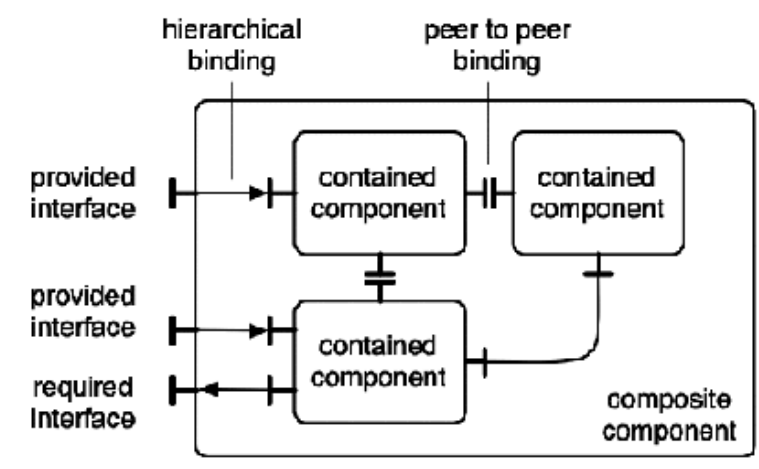

Figure 2. Composite Component on CBD

\section{Quality Measurement for Mobile M-ERP Applications}

\subsection{Our M-ERP Design Cponsiderations}

A mobile applications strategy for the enterprise is based on the following main considerations: Application functionality considerations, Technical considerations and Financial considerations The most important exercise is to identify the business areas of the organisation that will benefit the most from mobile applications and define the functionality of the applications. This requires a detailed analysis of the organisation's operations and its existing IT infrastructure. In the following paragraphs, we will give a 
general overview of enterprise business applications and the key areas suitable for mobile applications The front office applications cover the customer facing side of an organisation and are commonly called as Customer Relationship Management (CRM) systems.CRM systems provide applications for the sales force, call centre and customer service areas and provide functionality for taking orders, configuring complex products and providing effective service and support to customers. The customer facing professionals of an organisation (sales and service personnel) are typically mobile and the effectiveness of their activities depends a great deal on the timely and accurate access to information. Hence, applications for the mobile sales and service personnel of an organisation are the most suitable candidates for mobile applications. Some of these application areas are outlined below:

Sales Force Automation: Order Entry, Order Management, Lead Management, Point of Sale, Pricing and Configuration, Appointment and contact management Customer Support: Call Dispatch and Tracking, Customer Tracking, Technical Support Guides Field Service: Call Dispatch and Tracking, Logistics Management, Parts and Spares Management and Ordering, Parts Inventory Management, Field Manuals and Knowledge Tools.

There are some areas in back office application also, which can benefit from mobile applications. Some of the suitable back office application areas are: Asset Tracking, Inventory Management, Logistics Management, Order Entry, Procurement Management. After the application areas have been identified, the next major step is to identify the suitable technical solution. Various technical considerations are involved in choosing the right technical solution. Some of the main technical considerations are outlined below: Suitability for the Application: Does the technical solution have any unique capabilities to provide the features required by the application? Use of Existing IT Infrastructure: Does the solution make the best possible use of the existing IT infrastructure of the organisation? Integration with Existing Applications: How easy it is to integrate the mobile application with the existing M-ERP/SCM/CRM systems of the organisation?

Ease of Use: Simplicity of UI, ergonomics of the device.

Standards: Does the solution use the popular industry standards?

Connectivity: What means of wired and wireless connectivity are available in the territories covered by the application and which are supported by the proposed solution.

Upgrade Path: How is the technology for handheld devices and wireless connectivity going to evolve? How will the proposed solution upgrade to these advances?

Synchronisation: How will the data on the handheld device synchronise with the central data?

Development Tools: Are good development tools available for the platform?

Development Resources: Are software developers with skills in the chosen platform available?

Flexibility: How flexible is the solution. How easily can it be customised or changed?

Security: Are organisation's security requirements supported by the solution?

Scalability: How scalable is the solution with respect to the volume of data, number of users and number of transactions?

Reliability: What are the reliability requirements in quantitative terms? Does the solution support these requirements?

Ease of Management and Support: How easy is the management and support of the application. Can the management and support be done remotely?

Finally, any investment in technology needs to be justified by ROI considerations. A detailed financial analysis would be required for financial justification of an investment in mobile applications. However, we strongly believe that the benefits of the mobile 
applications are so compelling that ROI justification would be easily possible. The Figure 2 shows the process of Mobile ERP development in our study.

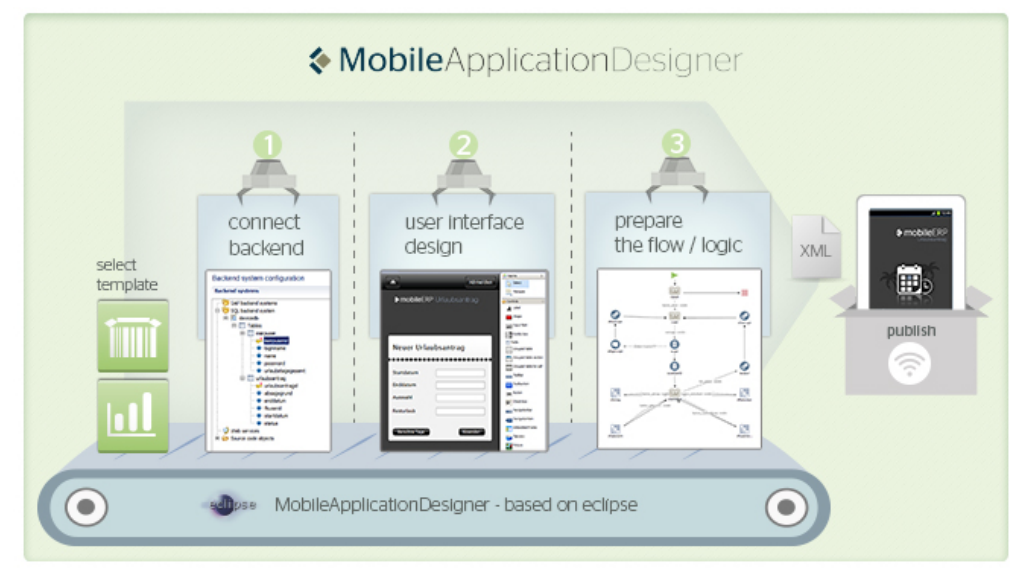

Figure 3. Process of Design for M ERP

\subsection{Our M-ERP Development Model}

The purpose of this study is to understand the critical role of the M-ERP system internal control, quality and performance in the factory industry. This paper examines the relationship of M-ERP system internal control, quality and performance. The Figure 3 sow the basic modules to develop the M ERP.

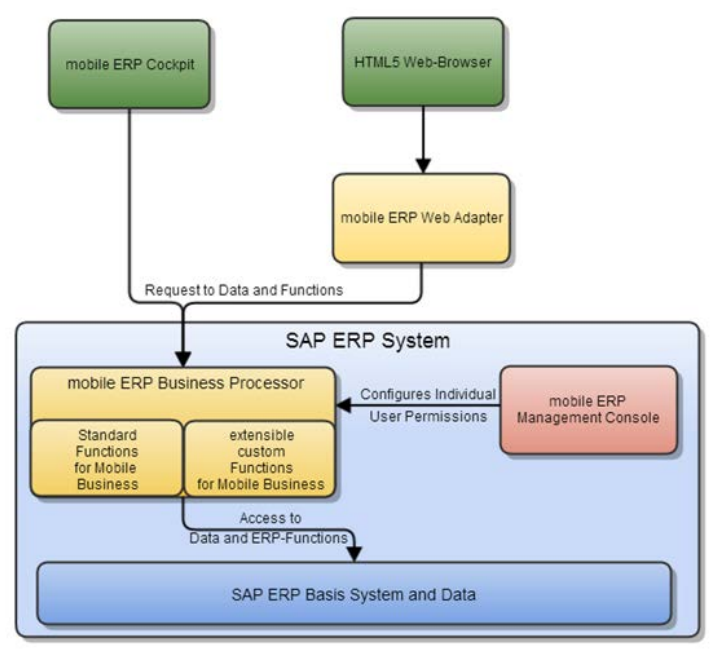

Figure 4. Our M ERP Modules

Via Internal Controls, the relevance and reliance to its objective has been more secure. Also enterprise information yielded by M-ERP System and efficiency of the information value has been considered very important. However, when it comes to contemporary studies, researches on M-ERP system Internal Controls and Quality, performance have not much been accumulated, so it is needed to be approached with diverse methods.

Therefore this paper set out the research model that the relationships of M-ERP system internal control, quality and performance (see Figure 4) 


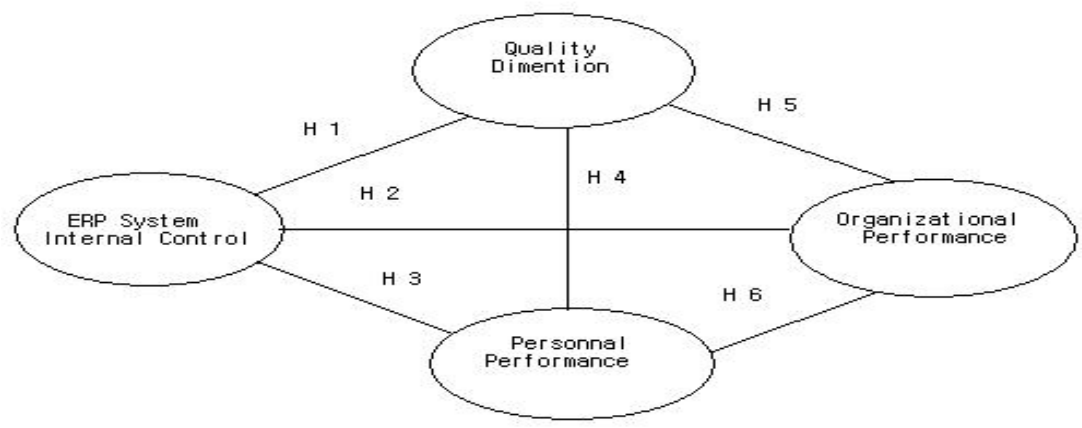

\section{Figure 5. Relationships of M-ERP System Internal Control, Quality and Performance}

To achieve this purpose, the following hypotheses are made.

$<$ Hypotheses 1(H1)> M-ERP system internal control will have a positive influence over quality dimension.

$<$ Hypotheses 2(H2)> M-ERP system internal control will have a positive influence over organizational performance.

$<$ Hypotheses 3(H3)> M-ERP system internal control will have a positive influence over personal performance.

$<$ Hypotheses 4(H4)> Quality dimension will have a positive influence over personal performance.

$<$ Hypotheses 5(H5)> Quality dimension will have a positive influence over organizational performance.

$<$ Hypotheses 6(H6)> Personal performance will have a positive influence over organizational performance.

\subsection{Our M-ERP Model Evaluation}

M-ERP system have been implemented and managed in order to deal with enterprise information more fast and precisely, and the suitability for the purpose and the reliance through Internal controls have been more ensured. And also products yielded by M-ERP system and the efficiency of the information value have been considered very important. But when it comes to existing studies, studies on internal controls and information quality which have an influence on the performance of M-ERP system are not much accumulated, so it is needed to approach with various methods. This study is for the purpose of understanding performance of M-ERP system more closely by means of perceiving relations between internal controls and information quality. That is, this study is to assess internal controls and information quality and to connect those with performance. This thesis is for the substantial study on whether information quality influenced by M-ERP system internal controls have an effect on performance, when internal controls of M-ERP system is good.

The relations between internal controls and information quality had statistical significance. The information quality has a statistically influence on the M-ERP systems' performance. And internal controls have a statistically influence on the M-ERP systems' performance. Through information quality, M-ERP system internal control will have a positive influence over performance. Consequently, the more investment in internal controls and quality, the greater the performance of the M-ERP systems. Therefore the higher the performance of the M-ERP systems through increased investment in internal control and information quality, the higher will be the value of the M-ERP system as a whole. 


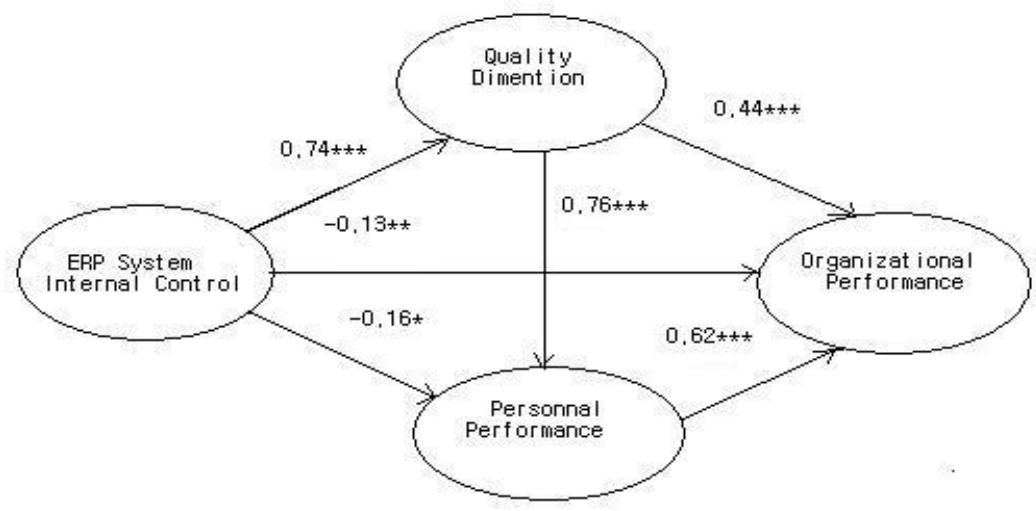

\section{Figure 5. M-ERP System Internal Control, Quality and Performance}

The first part involved a question about the respondent company' M-ERP internal control (planning \& organization, acquisition \& implementation, delivery \& support, monitoring). The second part involved a question about the respondent company' M-ERP quality domains (system quality, information quality, service quality). The third part consisted of the items measuring personal performance (usability, usefulness, satisfaction).

\section{Conclusion and Future Works}

Information technology has been playing a fundamental role in the factory industry for a long time. The Internet particularly is increasingly emerging as a perfect platform to bring enterprise information direct to the customer. Web-based M-ERP systems, however, are required not only to offer online brochures but also to provide both value and service. The M-ERP system meets this challenge by focusing on three crucial points. First, highquality access is provided, both by supporting e-commerce transactions and by allowing access to the system via the World Wide Web, info kiosks, and cellular phones. Second, the content is of a high quality that is achieved by enabling enterprise information providers to maintain their products directly, as well as by integrating external enterprise information sources. Finally, the M-ERP system is designed in such a way that it is easy to customize.

The purpose of this study is to understand the critical role of the M-ERP system's internal control, quality and performance in the factory industry. Therefore this paper examines the relationships of the M-ERP system's internal control and quality dimension on performance. This sets out the research model of influencing factors and the performance of the M-ERP system through theoretical studies based on Management Information Systems, and the empirically tests hypotheses related to the model. As the result of the study, the relations between internal controls and quality dimension has statistical significance. The quality dimension has a statistically influence on the personal performance and organizational performance. Through path analysis, this study examined the influence which relations between internal controls and quality have on performance and is very meaningful in terms of the first trial. And on the point of time when there are little studies on the effect analyses among internal controls, quality dimension, performance, this study would be the promoter for the future. The limitation on this study is to analyze only mutual effects between factors by choosing internal controls, quality dimension and performance as survey variables. The study which measure items with precision which are related to each variable and understand measurement factors clearly should be implemented. Although this paper has already established a research framework, as exploratory research, it must also take the next step of refining, leading to the guide for future research work. This study is the first study to analyze comprehensively the 
relationship of the internal control, quality dimension on the M-ERP system performance, therefore further studies using generalized research methodology are recommended. And a longitudinal research design is essential to confirm the linkages among the study variables.

\section{Acknowledgment}

"This research was also supported by the International Research \& Development Program of the National Research Foundation of Korea (NRF) funded by the Ministry of Science, ICT \& Future Planning (Grant number: K 2012057499)”.

\section{References}

[1] A. Brown, "Using service-oriented architecture and component-based development to build Mobile service applications”, Rational Software white paper from IBM, (2002) April.

[2] R. Soley and OMG Staff Strategy Group, "Model Driven Architecture”, OMG Whit Paper Draft 3.2, at URL: http://www.omg.org/ soley/mda.html, (2000).

[3] J. D. Poole, "Model Driven Architecture: Vision, Standards and Emerging Technologies", European Conference on Object-Oriented Programming, at URL: http://www.omg.org/mda/mda_files/ModelDriven_Architecture.pdf, (2004) April.

[4] M. Rizwan Jameel Qureshi, "Reuse and Component Based Development”, Proc. of Int. Conf. Software Engineering Research and Practice (SERP'06 Las Vegas, USA), (2006) June 26-29, pp. 146-150.

[5] A. Barnawi, M. Rizwan Jameel Qureshi and A. Irshad Khan, "A Framework for Next Generation Mobile and Wireless Networks Application Development using Hybrid Component Based Development Model”, International Journal of Research and Reviews in Next Generation Networks (IJRRNGN), vol. 1, no. 2, (2011) December, pp. 51-58.

[6] M. Champion, C. Ferris, I. E. Newcomer and D. Orchard, "Mobile Services Architecture: W3C Working Draft”, http://www.w3.org/TR/ws-arch/, (2012). 
International Journal of Smart Home

Vol.7, No.6 (2013) 